九州大学学術情報リポジトリ

Kyushu University Institutional Repository

\title{
Surface Relaxation of W(110) and 2D Growth of B Studied by Low Energy Electron Diffraction and Auger Electron Spectroscopy
}

\section{Shahadat Hossain}

Department of Advanced Energy Engineering Science, Interdisciplinary Graduate School of Engineering Science, Kyushu University

Farhana Jesmin Tuli

Department of Advanced Energy Engineering Science, Interdisciplinary Graduate School of Engineering Science, Kyushu University

\section{Peng Guansong}

Department of Advanced Energy Engineering Science, Interdisciplinary Graduate School of Engineering Science, Kyushu University

\section{Nakagawa, Takeshi}

Department of Advanced Energy Engineering Science, Interdisciplinary Graduate School of Engineering Science, Kyushu University

他

https://doi.org/10.5109/4102456

出版情報: Proceedings of International Exchange and Innovation Conference on Engineering \& Sciences (IEICES). 6, pp.7-13, 2020-10-22. Interdisciplinary Graduate School of Engineering Sciences, Kyushu University バージョン：

権利関係 : 


\title{
Surface Relaxation of W(110) and 2D Growth of B Studied by Low Energy Electron Diffraction and Auger Electron Spectroscopy
}

\author{
Shahadat Hossain ${ }^{1,2, *}$, Farhana Jesmin Tuli ${ }^{1}$, Peng Guansong ${ }^{1}$, Takeshi Nakagawa ${ }^{1}$, Seigi Mizuno ${ }^{1}$ \\ ${ }^{1}$ Department of Molecular and Material Sciences, Interdisciplinary Graduate School of Engineering Sciences, \\ Kyushu University, Fukuoka, Japan. \\ ${ }^{2}$ Atomic Energy Centre, Bangladesh Atomic Energy Commission, Chattogram, Bangladesh. \\ *Corresponding author email: shahadat.baec@gmail.com
}

\begin{abstract}
Here we report the growth of elemental $B$ on $W(110)$ surface by low energy electron diffraction and Auger electron spectroscopy study with multilayer relaxation of W(110) surface. Our study reveals that, first interlayer spacing of $\Delta d_{12} / d=-1.2 \pm 1.5 \%$ causes an expansion in the second and third interlayer spacing of $\Delta d_{23} / d$ $=0.7 \pm 1.5 \%$ and $\Delta d_{34} / d=0.5 \pm 2.6 \%$, respectively in $W(110)$. And we have obtained a Pendry $R_{p}=0.20$ for $W(110)$. Boron produces a $c(9 \times 1)$ structure on $W(110)$ after annealing at $1000{ }^{\circ} \mathrm{C}$ for 10 minutes which is sustainable up to $1300{ }^{\circ} \mathrm{C}$. These results will pave the way for surface structure determination of $B$ on W(110).
\end{abstract}

Keywords: Surface relaxation; Tungsten; Boron; LEED; AES.

\section{INTRODUCTION}

An interest in low-dimensional structures has snowballed in the past decade with the flourishing of graphene [1]. Due to the inimitable physical and chemical properties, plenty of other two-dimensional (2D) materials have also been synthesized or theoretically predicted such as silicene [2,3], germanene [4], phosphorene [5], arsenene [6] and borophene [7-14], which are the neighboring elements of carbon in the periodic table. These 2D materials show enormous application prospect in electronic and spintronic devices, energy storage and utilization. However, the synthesis of elemental 2D phases has been proven to be much more difficult [8]. Among them, boron is of great interest and challenging as borophene can show metallicity in the majority of phases, high mechanical flexibility and strength, optical transparency, magnetism, superconductivity and the ability to host Dirac cones [15].

Substrates for the 2D materials play important roles as the interaction between them is complex. It also affects the structural and electronic properties along with the practicability of their preparation. A large number of theoretical works were done finding the stable 2D boron phases like $\alpha$ sheet, $\beta$ sheet and $\chi$ sheet in several substrates, but very few experimental realizations have been achieved only recently. The first experimental synthesis of borophene was reported independently by two groups where two distinct phases $\left(\beta_{12}\right.$ and $\left.\chi_{3}\right)$ coexisted on $\operatorname{Ag}(111)$ substrate $[7,8]$. Another report on the same substrate claimed to find some more phases (hexagonal $\alpha$ sheet and differently rotated variants of $\beta_{12}$ ) including the previous ones [9]. This group has also reported four phases ( 2 mirror-imaged $\beta$ boron sheet, $\beta_{8}$ and $\chi_{3}$ ) of borophene nanoribbons on $\operatorname{Ag}(110)$ surface [16]. It was assumed that $\mathrm{Ag}$ would be the best metallic substrate for borophene growth because it is not likely to boride formation but dynamic enough to stabilize borophene. However, there is existence of several phases due to comparatively weak interaction between boron and silver. Multiple phases can't be omitted by controlling the growth temperature as well. This is a significant problem from the application standpoint. Very recently borophene was successfully prepared on some other substrates, e.g., $\mathrm{Cu}(111)$ [10], $\mathrm{Al}(111)$ [11], $\mathrm{Au}(111)$ [13] and $\operatorname{Ir}(111)$ [14] where boron honeycomb structure $\left(\delta_{3}\right.$ phase) was formed on $\mathrm{Al}(111), \alpha^{\prime}$-borophene on $\mathrm{Au}(111)$ and $\chi_{6}$ on $\operatorname{Ir}(111)$. So far, the reported growth processes, chemical bonding, electronic states and temperature regimes are significantly diverse for borophene grown on metal substrates. Optimized conditions for preparing stable 2D boron phases for practical application need more study using new substrates.

$\mathrm{W}(110)$ is the most stable surface of tungsten and could be a possible candidate for $2 \mathrm{D}$ boron growth. This surface showed steadiness and outstanding performance in growth of carbon nanotubes [17]. One theoretical work has also reported the formation of unique $2 \mathrm{D}$ boron sheet (named as $\pi$ phase) on W(110), which can retain its structure upon exfoliation and stable against high temperatures (up to $1800 \mathrm{~K}$ ). Also, this phase of boron possesses special electronic structure and anisotropic electric conductivity, which 
can be of great interest for application in electronic devices [18]. Being stimulated with the possibility of forming stable borophene on $\mathrm{W}(110)$, therefore, in this work we have used the $\mathrm{W}(110)$ surface as a substrate for $2 \mathrm{D}$ growth of boron.

To analyze the structure of adsorbate-covered crystal surface, it is important to have the idea about the structure of clean surface as the atomic arrangements of first few layers have a profound relation to their physical, chemical and catalytic properties [19]. Generally, the $1^{\text {st }}\left(d_{12}\right)$ and $2^{\text {nd }}\left(d_{23}\right)$ normal interlayer spacing are compressed and expanded relative to their bulk, respectively. Still, inconsistencies occur frequently between different investigations with respect to the magnitude of the relaxation. Similarly, there are disagreements in surface relaxation studies of $\mathrm{W}(110)$. Still it is not clear whether they arise from experimental errors or theoretical methodology or both. Therefore, we tried to study the interlayer relaxation or surface structure of clean W(110) first by minimizing the experimental errors as much as possible. Later we tried to optimize the growth condition of B on W(110) surface by low energy electron diffraction (LEED) and Auger electron spectroscopy (AES). This study will shed light on 2D boron growth on $\mathrm{W}(110)$. Our future endeavor will include the surface structure of B on W(110) surface.

\section{EXPERIMENTAL}

The chamber used to carry out the measurements was equipped with four grid LEED system, AES, e-beam evaporator, quartz crystal microbalance (QCM) and pressure gauge. After bakeout and outgassing, ultrahigh vacuum (UHV) environment with a base pressure of $2 \times 10^{-8} \mathrm{~Pa}$ was ensured to perform the experiments. The $\mathrm{W}(110)$ substrate was cleaned by repeatedly annealing in $6 \times 10^{-6} \mathrm{~Pa}$ oxygen atmosphere at $1200{ }^{\circ} \mathrm{C}$ for 10 minutes to deplete near-surface carbon. Any residual oxygen detected after annealing was removed by flashing the sample at $1800{ }^{\circ} \mathrm{C}$ for a few seconds. This procedure resulted in a surface free of contaminants, which was later confirmed by AES. After cleaning, we used liquid $\mathrm{N}_{2}$ to cool the sample. At a temperature of $120 \mathrm{~K}$, the LEED patterns were measured using a digital charge-coupled device (DCCD) camera with a computer-controlled data acquisition system. The temperature was measured by means of a thermometer. Incident energy range for LEED was $100-500 \mathrm{eV}$. The data sets for $I(E)$ spectra were obtained after symmetrizing (symmetrydegenerate) conjugate beams in the usual manner.
The orientation of the sample was adjusted so that all conjugate beams show the same $I(E)$ shape.

Pure B was evaporated from a crucible using e-beam evaporator and deposited on cleaned W(110) surface at room temperature. The deposition rate of $\mathrm{B}$ was monitored with QCM. Several samples were prepared by varying the deposition amount of $\mathrm{B}$ on $\mathrm{W}(110)$ surface and the samples were annealed at different temperatures (ranging from room temperature to 1500 ${ }^{\circ} \mathrm{C}$ ) for 10 minutes. LEED patterns for several condition was taken and $I(E)$ spectral data for optimum condition was also recorded as described before.

\section{STRUCTURAL CALCULATION}

We used a Barbieri/Van Hove symmetrized automated tensor LEED package for calculating the theoretical $I(E)$ curves for structure models to specify the atomic positions [20]. The imaginary part of the inner potential $\left(V_{o i}\right)$ was fixed to $-5.0 \mathrm{eV}$ but the real part was determined through theory-experiment fit. The best-fit model was obtained based on the best agreement between the experimental and theoretical $I(E)$ curves, which was decided by minimizing Pendry's reliability factor $\left(R_{p}\right)$ [21]. The error bar in structural parameters was calculated by variance of $R_{p}$,

$$
\Delta R=R_{\min }\left(8\left|V_{o i}\right| / \Delta E\right)^{1 / 2}
$$

where $R_{\min }$ is the lowest $R_{p}$ value and $\Delta E$ is the total energy range of the experimental $I(E)$ curves [21].

\section{RESULTS AND DISCUSSIONS \\ 4.1 Surface relaxation of clean $W(110)$}

An atomically clean W(110) surface was observed by LEED after successive annealing in oxygen atmosphere and flashing at high temperature. Fig. 1 shows the LEED pattern of clean W(110) surface which reflects the $\mathrm{p}(1 \times 1)$ (marked by red diamond) structure.

Initially, structure of the clean W(110) surface at room temperature was determined by tensor LEED. Fig. 2 illustrates the comparison between best-fit and experimental spectra for six beams: $(1,0),(1,1),(1,-$ $1),(2,0),(2,1)$ and $(2,-1)$. The present LEED $I(E)$ spectra demonstrates a multilayer relaxation in the $\mathrm{W}(110)$ surface which is similar to the previous studies [22-25]. This was achieved by obtaining the minimum Pendry $R_{p}=0.20$ which is comparable with previous study $\left(R_{p}=0.202[23]\right)$. 


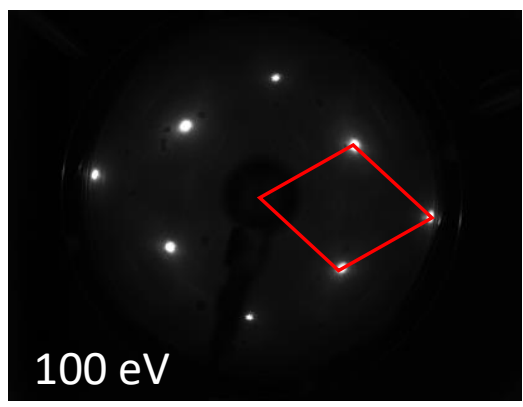

Fig. 1. A LEED pattern for clean W(110) surface after successive annealing $\left(6 \times 10^{-6} \mathrm{~Pa}\right.$ oxygen atmosphere at $1200{ }^{\circ} \mathrm{C}$ for 10 minutes) and flashing $\left(1800{ }^{\circ} \mathrm{C}\right)$.

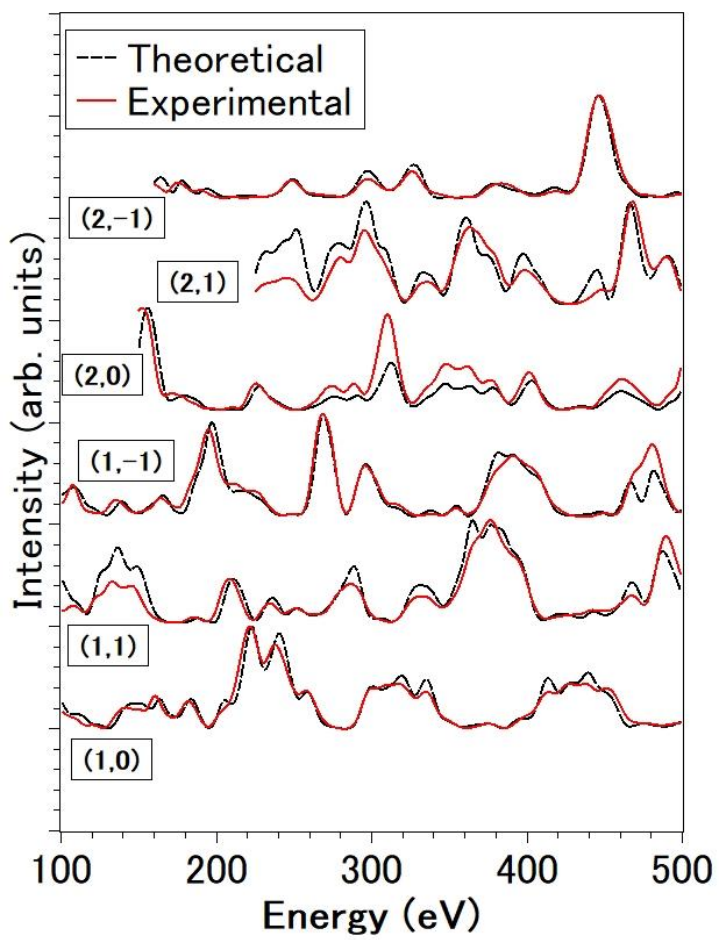

Fig. 2. Best-fit comparison between experimental and theoretical LEED $I(E)$ spectra for clean W(110).

The optimized Debye temperatures for $\mathrm{W}$ in the first layer and in the next layers were 130, 245, 300 and $310 \mathrm{~K}$, respectively. The sphere model of surface atoms is shown in Fig. 3. Comparing the bulk interlayer spacing value $d=2.24 \AA$, it is shown here that a contraction in the first interlayer spacing of $\Delta d_{12} / d=-1.2 \pm 1.5 \%$ causes an expansion in the second and third interlayer spacing of $\Delta d_{23} / d=0.7 \pm$ $1.5 \%$ and $\Delta d_{34} / d=0.5 \pm 2.6 \%$, respectively.

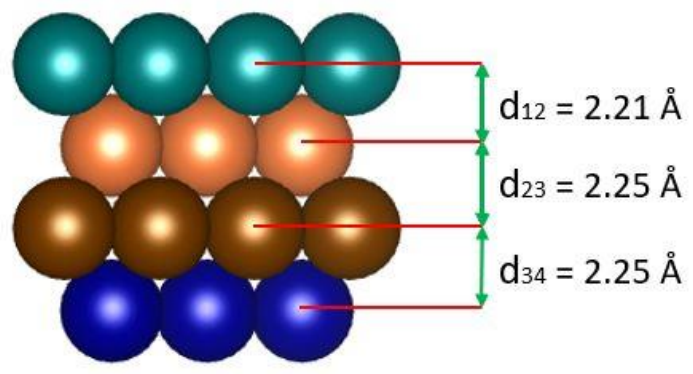

Fig. 3. Surface relaxation in W(110) (side view).

Earlier theoretical works found values for $\Delta d_{12} / d$ ranging from $-1.4 \%$ to $-5 \%$ and for $\Delta d_{23} / d$ from $-0.4 \%$ to $4.6 \%$ [22,26-29]. The structural parameters (both $\Delta d_{12} / d$ and $\Delta d_{23} / d$ ) of the present work are slightly different from those of earlier LEED studies. However, if we consider the variance of $R_{p}$, the present result is also comparable with them. One major difference between the earlier works and our study is the size of the data set analyzed. Our structure analysis is based on LEED $I(E)$ spectra from six inequivalent beams to an energy of $500 \mathrm{eV}$ which corresponds to a data set covering an equivalent range of about $2400 \mathrm{eV}$. It is also seen that $R_{p}$ decreases as the energy cutoff (size of the data set) increases [23]. Additionally, it was also suggested that the surface roughness could be the possible reason of the inconsistencies between surface structure determined from ab initio calculations and LEED measurements.

\subsection{Growth of $B$ on W(110) 4.2.1 LEED pattern analysis}

With the deposition of B onto W(110) surface at room temperature, the diffraction pattern remained almost the same as the clean surface, which depicts the "amorphous" form of B. When the coverage of B increased, the background became more diffused. This background diffusion might be due to the disordered state of $\mathrm{B}$ on $\mathrm{W}(110)$. The samples covered with B were annealed at different temperatures ranging from room temperature to 

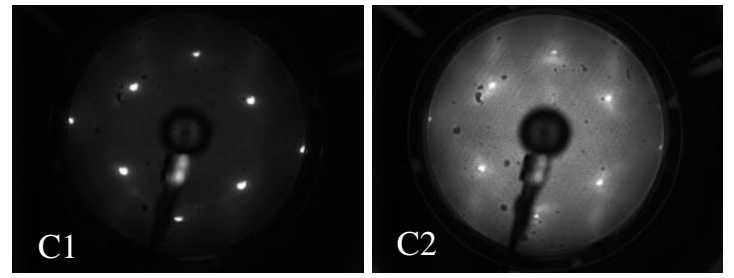

(a)
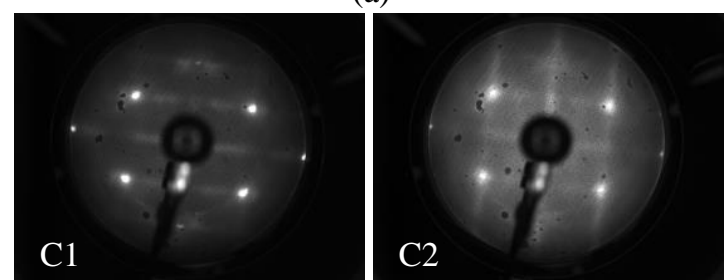

(b)

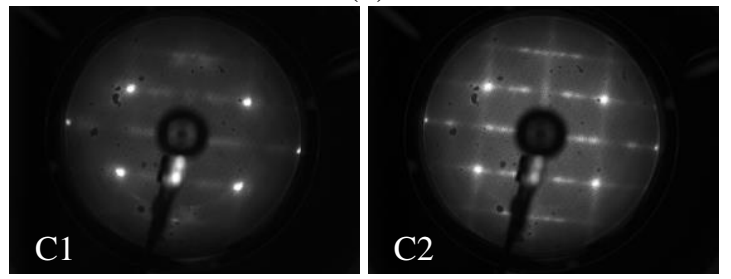

(c)

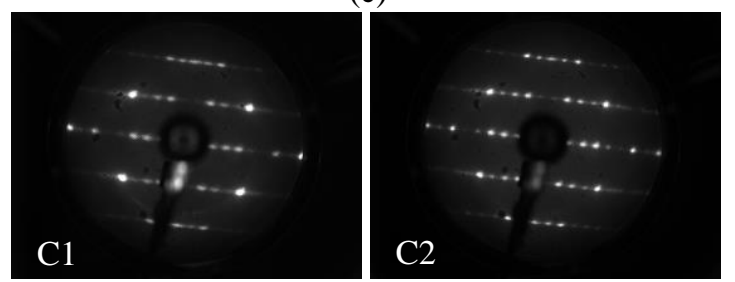

(d)

Fig. 4. LEED pattern changes at different annealing temperatures $\left(\mathrm{E}_{\mathrm{p}}=100 \mathrm{eV}\right)$. (a) $500{ }^{\circ} \mathrm{C}$, (b) $700{ }^{\circ} \mathrm{C}$, (c) $900{ }^{\circ} \mathrm{C}$, (d) $1100{ }^{\circ} \mathrm{C}$.

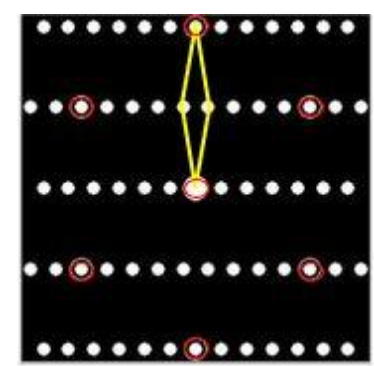

Fig. 5. Schematic LEED pattern for B/W(110). The yellow diamond denotes the corresponding reciprocal unit cell.

$1500{ }^{\circ} \mathrm{C}$. There were no prominent changes in the LEED pattern below $500{ }^{\circ} \mathrm{C}$. So, here we discuss the annealing effect from $500{ }^{\circ} \mathrm{C}$ to $1500{ }^{\circ} \mathrm{C}$ for 10 minutes. We can describe the annealing effect by dividing the samples into two categories.

The first category $(\mathrm{C} 1)$ includes the samples with low B deposition time (5 $\mathrm{min}$ to $20 \mathrm{~min}$ ) and the second category (C2) includes samples with relatively higher B deposition time (30 min to $60 \mathrm{~min}$ ). Fig. 4 shows LEED pattern changes depending on the annealing temperatures. With annealing at $500{ }^{\circ} \mathrm{C}$, the $\mathrm{C} 1$ shows no major changes and the $\mathrm{C} 2$ shows broad and weak diffusion as shown in Fig. 4(a). The samples were then annealed at $700{ }^{\circ} \mathrm{C}$ and the corresponding LEED patterns are shown in Fig. 4(b). In this step, C1 samples started to show streak between integer spots and for C2 samples the broad and weak spots continued. The samples were further annealed at 900 ${ }^{\circ} \mathrm{C}$ and the corresponding LEED patterns are shown in Fig. 4(c). This step is assumed to be a prolongation of the previous step for $\mathrm{C} 1$ samples. For $\mathrm{C} 2$ samples, an imperfect long-range ordering was observed. B might have started to form 2D growth in this step.

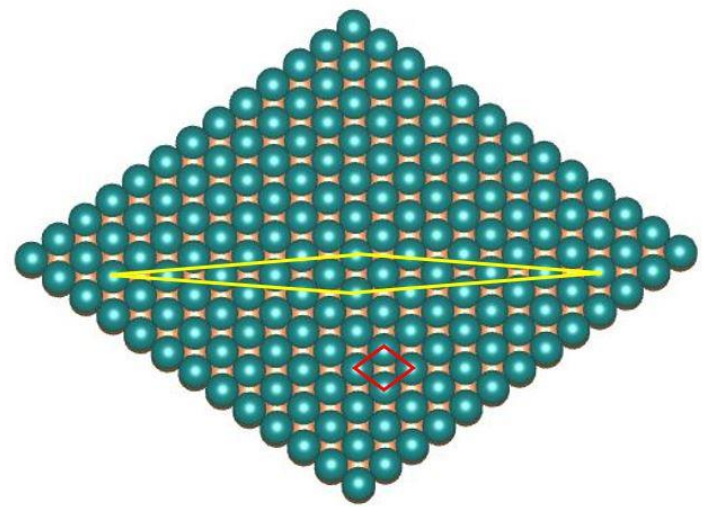

Fig. 6. Real space visualization of unit cell for $\mathrm{B} / \mathrm{W}(110)$ (yellow diamond) and clean $\mathrm{W}(110)$ (red diamond).

When the samples were annealed at $1100{ }^{\circ} \mathrm{C}$, an ordered structure appeared, which is shown in Fig. 4(d). Following Wood's notation, the phase can be described as $\mathrm{c}(9 \times 1)$ structure for $\mathrm{B} / \mathrm{W}(110)$ as described in Fig. 5. A real space view of the unit cell is shown in Fig. 6. Again, annealing samples at 1200 ${ }^{\circ} \mathrm{C}$ have given a more clearer LEED pattern for $\mathrm{C} 2$ samples. Still the samples show the same structure. Afterwards, the samples were annealed at $1300{ }^{\circ} \mathrm{C}$, where the $\mathrm{c}(9 \mathrm{x} 1)$ pattern got weaker. With further 
annealing at $1500{ }^{\circ} \mathrm{C}$, the $\mathrm{p}(1 \times 1)$ structure was formed. These changes in LEED pattern confirms the disappearance of $\mathrm{B}$ atoms from the $\mathrm{W}$ surface due to the diffusion of $\mathrm{B}$ atoms into bulk $\mathrm{W}$ crystal or evaporation from the surface.

\subsubsection{AES analysis}

We performed AES measurements to understand the chemical environment and growth of the $\mathrm{B} / \mathrm{W}(110)$. Because both $\mathrm{W}$ and $\mathrm{B}$ show strong peaks around 179 $\mathrm{eV}$ as shown in Fig. 7, it is tough to differentiate them. Representative AES spectra was obtained for clean $\mathrm{W}(110)$, after B deposition and at different annealing conditions. In the clean $\mathrm{W}(110)$ spectrum, a sharp peak was seen at $179 \mathrm{eV}$ which is the characteristic $\mathrm{W}$ spectrum and no extra peaks were observed due to contaminants or oxygen (around $500 \mathrm{eV}$ ) [30]. Fig. 8 shows the corresponding intensity variation of the AES peak at $179 \mathrm{eV}$. After boron deposition, the peak intensity at $179 \mathrm{eV}$ increased noticeably. With annealing the sample up to $700{ }^{\circ} \mathrm{C}$ the peak intensity did not change drastically. However, with annealing the sample at $900{ }^{\circ} \mathrm{C}$, the peak intensity drastically increased. With the further increase of the annealing temperature, the peak intensity started to decrease.

The AES spectra of the $\mathrm{B} / \mathrm{W}(110)$ is analogous to the LEED pattern observed at different annealing conditions. Initially after B deposition on W(110) surface, B formed 3D island and did not cover the W substrate completely. After annealing the sample, B island changes its morphology and covers the substrate more. This morphological change happened around $900{ }^{\circ} \mathrm{C}$. When the sample was annealed at 900 ${ }^{\circ} \mathrm{C}$, it has started to grow in the ordered 2D structure, which we also could see in LEED pattern (Fig. 4). After that, annealing the sample gave much clearer $\mathrm{c}(9 \times 1)$ superstructure, which sustained up to $1300{ }^{\circ} \mathrm{C}$. The AES intensity also remained comparable between $900{ }^{\circ} \mathrm{C}$ to $1300{ }^{\circ} \mathrm{C}$. The peak intensity declined rapidly after heating the sample at $1400{ }^{\circ} \mathrm{C}$ or more, which also matched well with LEED pattern as the superstructure disappeared at this region. According to our study, B atoms form 2D structure on $\mathrm{W}(110)$ after annealing at $1000{ }^{\circ} \mathrm{C}$, which can sustain up to $1300{ }^{\circ} \mathrm{C}$.

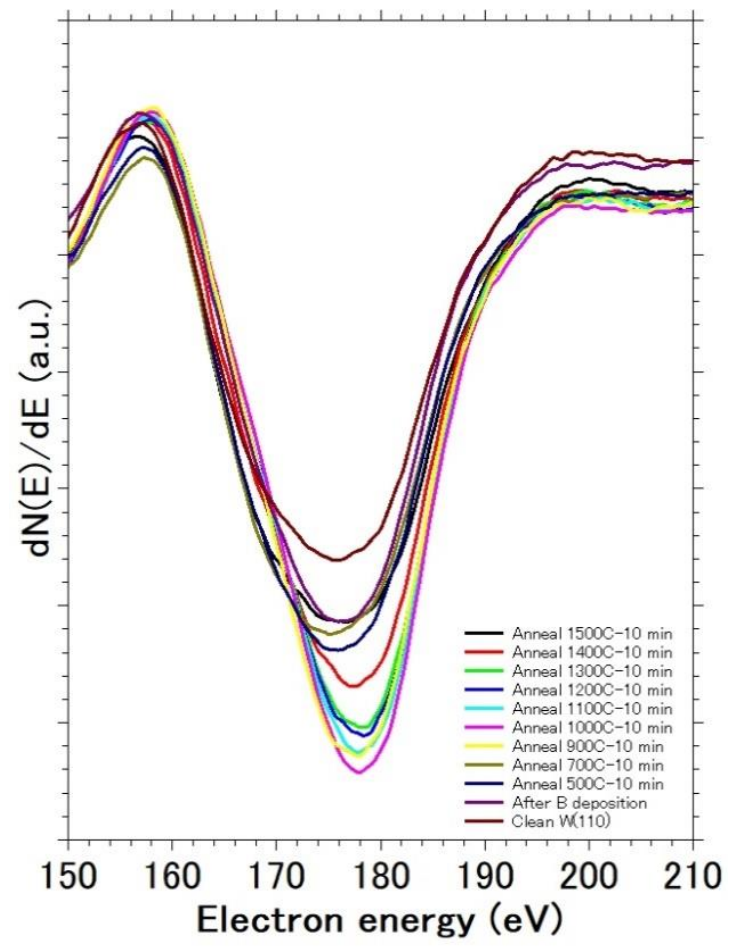

Fig. 7. AES spectra for $\mathrm{B} / \mathrm{W}(110)$ at different annealing conditions.

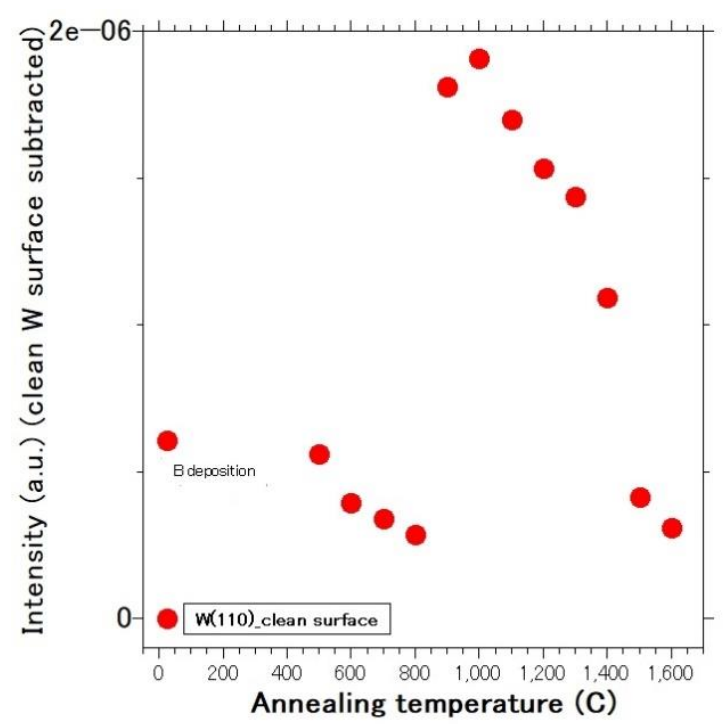

Fig. 8. AES intensity variation with annealing temperature.

\section{CONCLUSIONS}

We have studied the multilayer surface relaxation of $\mathrm{W}(110)$ by LEED $I(E)$ analysis. The study reveals that 
a contraction in the first interlayer spacing of $\Delta d_{12} / d=$ $-1.2 \pm 1.5 \%$ which is responsible for an expansion in the second and third interlayer spacing of $\Delta d_{23} / d=0.7$ $\pm 1.5 \%$ and $\Delta d_{3} / d=0.5 \pm 2.6 \%$, respectively. Also, we have found the Pendry $R_{p}=0.20$. Our results are in good agreement with previous studies if we consider the variance. It is noteworthy that we increased our data set to an equivalent range of 2400 $\mathrm{eV}$ and number of analyzed beams to six to minimize the experimental errors as much as possible.

We have also studied the growth of B on W(110) surface by LEED and AES. It is seen that B does not produce any crystal structure after immediate deposition of it on $\mathrm{W}(110)$ surface and annealing up to $800{ }^{\circ} \mathrm{C}$ due to its amorphous nature. Rather it produces $\mathrm{c}(9 \times 1)$ unit cell on $\mathrm{W}(110)$ when it is annealed at $1000{ }^{\circ} \mathrm{C}$. This phase of B sustains up to $1300{ }^{\circ} \mathrm{C}$. AES study is also similar to the LEED studies. This creates the possibilities of borophene growth on W(110) as predicted by earlier theoretical work. Our future work involves the structure determination of the prepared sample.

\section{REFERENCES}

[1] A. K. Geim, K. S. Novoselov, The rise of graphene, Nat. Mater. 6 (2007) 183-191.

[2] A. Molle, C. Grazianetti, L. Tao, D. Taneja, M.H. Alam, D. Akinwande, Silicene, silicene derivatives, and their device applications, Chem. Soc. Rev. 47 (2018) 6370.

[3] G. Li, L. Zhang, W. Xu, J. Pan, S. Song, Y. Zhang, H. Zhou, Y. Wang, L. Bao, Y.Y. Zhang, S. Du, M. Ouyang, S.T. Pantelides, H.J. Gao, Stable Silicene in Graphene/Silicene Van der Waals Heterostructures, Adv. Mater. e1804650 (2018).

[4] G. Liu, S.B. Liu, B. Xu, C.Y. Ouyang, H.Y. Song, S. Guan, S.A. Yang, Multiple Dirac points and hydrogenation-induced magnetism of germanene layer on $\mathrm{Al}$ (111) surface, J. Phys. Chem. Lett. 6 (2015) 4936.

[5] X.R. Hu, J.M. Zheng, Z.Y. Ren, Strong interlayer coupling in phosphorene/graphene van der Waals heterostructure: A first-principles investigation, Front. Phys. 13(2) (2018) 137302.

[6] S. Zhang, Z. Yan, Y. Li, Z. Chen, and H. Zeng, Atomically thin arsenene and antimonene: semimetal-semiconductor and indirect-direct band-gap transitions, Angew Chem. Int. Edit. 54 (2015) 3112.

[7] A. J. Mannix, X.-F. Zhou, B. Kiraly, J. D. Wood, D. Alducin, B. D. Myers, X. Liu, B. L. Fisher, U. Santiago, J. R. Guest, M. J. Yacaman, A. Ponce, A. R. Oganov, M. C. Hersam, N. P. Guisinger, Synthesis of borophenes: Anisotropic, twodimensional boron polymorphs, Science 350(6267) (2015) 1513-1516.

[8] B. Feng, J. Zhang, Q. Zhong, W. Li, S. Li, H. Li, P. Cheng, S. Meng, L. Chen, K. Wu, Experimental realization of two-dimensional boron sheets, Nat. Chem. 8 (2016) 563-568.

[9] Q. Zhong, J. Zhang, P. Cheng, B. Feng, W. Li, S. Sheng, H. Li, S. Meng, L. Chen, K. Wu, Metastable phases of 2D boron sheets on Ag(111), J. Phys.: Condens. Matter 29 (2017) 095002.

[10] R. Wu, I. K. Drozdov, S. Eltinge, P. Zahl, S. Ismail-Beigi, I. Bozovic, A. Gozar, Large-area single-crystal sheets of borophene on $\mathrm{Cu}(111)$ surfaces, Nat. Nanotechnol. 14 (2019) 44-49.

[11] W. Li, L. Kong, C. Chen, J. Gou, S. Sheng, W. Zhang, H. Li, L. Chen, P. Cheng, K. Wu, Experimental realization of honeycomb borophene, Sci. Bull. 63 (2018) 282-286.

[12] L. Zhu, B. Zhao, T. Zhang, G. Chen, S. A. Yang, How is honeycomb borophene stabilized on Al(111)? J. Phys. Chem. C 123 (2019) 14858.

[13] B. Kiraly, X. Liu, L. Wang, Z. Zhang, A. J. Mannix, B. L. Fisher, B. I. Yakobson, M. C. Hersam, N. P. Guisinger, Borophene synthesis on $\mathrm{Au}(111)$, ACS Nano 13 (2019) 3816-3822.

[14] N. A. Vinogradov, A. Lyalin, T. Taketsugu, A. S. Vinogradov, A. Preobrajenski, Single-phase borophene on $\operatorname{Ir}(111)$ : Formation, structure and decoupling from the support, ACS Nano 13 (2019) 14511-14518.

[15] Z. Q. Wang, T. Lu, H. Wang, Y. P. Feng, J. Zheng, Review of borophene and its potential applications, Front. Phys. 14(2) (2019) 23403.

[16] Q. Zhong, L. Kong, J. Gou, W. Li, S. Sheng, S. Yang, P. Cheng, H. Li, K. Wu, L. Chen, Synthesis of borophene nanoribbons, Phys. Rev. Mater. 1 021001(R) (2017) 1-5.

[17] F. Yang, X. Wang, D. Zhang, J. Yang, D. Luo, Z. Xu, J. Wei, J.-Q. Wang, Z. Xu, F. Peng, Chirality-specific growth of single-walled 
carbon nanotubes on solid alloy catalysts, Nature 510 (2014) 522-524.

[18] Z. Cui, E. Jimenez-Izal, A. N. Alexandrova, Prediction of two-dimensional phase of boron with anisotropic electric conductivity, J. Phys. Chem. Lett., 8 (2017), 1224-1228.

[19] C. B. Duke (Ed.), Surface Science: the first thirty years, Surf. Sci. 299/300 (1994) 1.

[20] M. A. Van Hove, W. Moritz, H. Over, P. J. Rous, A. Wander, A. Barbieri, N. Materer, U. Strarke, G. A. Somorjai, Automated determination of complex surface structures by LEED, Surf. Sci. Rep., 19 (1993) 191-229.

[21] J.B. Pendry, Reliability factors for LEED calculations, J. Phys. C: Solid State Physics, 13 (1980) 937.

[22] M. Arnold, G. Hupfauer, P. Bayer, L. Hammer, K. Heinz, B. Kohler, M. Scheffler, Hydrogen on W(110): an adsorption structure revisited, Surf. Sci. 382 (1997) 288-299.

[23] G. Teeter, J. L. Erskine, Surface roughness and LEED crystallography: Analysis of flat and vicinal W(110), Phys. Rev. B 60(3) (1999) 19751981.

[24] D. Venus, S. Cool, M. Pilhal, Quantitative structural determination using spin-polarized low-energy electron diffraction rotation curves: W(110) Surf. Sci. 446(3) (2000) 199-210.

[25] H. L. Meyerheim, D. Sander, R. Popescu, P. Steadman, S. Ferrer, J. Kirschner, Interlayer relaxation of $\mathrm{W}(110)$ studied by surface $\mathrm{X}$-ray diffraction, Surf. Sci. 475 (2001) 103-108.

[26] J. S. Luo, B. Legrand, Multilayer relaxation at surfaces of body-centered-cubic transition metals, Phys. Rev. B 38(3) (1988) 1728-1733.

[27] A. M. Rodrigquez, G. Bozzolo, J. Ferrante, Multilayer relaxation and surface energies of fcc and bcc metals using equivalent crystal theory, Surf. Sci. 289 (1993) 100-126.

[28] W. Xu, J. B. Adams, W single adatom diffusion on W surfaces, Surf. Sci. 319 (1994) 58-67.

[29] X. Qian, W. Hubner, First-principles calculation of structural and magnetic properties for $\mathrm{Fe}$ monolayers and bilayers on W(110), Phys. Rev. B 60(23) (1999) 16192-16197.

[30] Handbook of Auger Electron Spectroscopy: A book of reference data for identification and interpretation in Auger electron spectroscopy, Physical Electronics, Chanhassen, MN, 1995. 